\title{
Design of A Micro-Cantilever Biosensor for Micro-Level Detections
}

\author{
Meher Dev Gudea \\ Department of Mechanical Engineering \\ Dwarkadas J. Sanghvi College of Engineering \\ Mumbai, India
}

\author{
Atharv Kulkarni \\ Department of Mechanical Engineering \\ Lokmanya Tilak College of Engineering \\ Navi Mumbai, India
}

\begin{abstract}
A modern approach for the creation of nanomechanical-based biosensors is the surface immobilization of functional receptors in microfabricated cantilevers arrays. Microcantilever-based systems can identify unlabeled diseases in very limited quantities of samples in real-time and multiplexed ways. The processing technologies presently available would enable the incorporation and analysis of one piece of equipment, reducing the size of the device, detection time, and expenses. Microfabricated cantilever array-based biosensing systems, requiring numerous cantilevers, electronic processing, and even local telemetry on a single device, can address the extremely responsive and limited multi-target detection needs for very small samples. Here we discuss the design and development cycle of biosensors based on the cantilever.
\end{abstract}

Keywords- Biomechanics; Cantilever Array; Biosensors; Piezoresistivity; Micro-Level Detection;

\section{INTRODUCTION}

Multidisciplinary work is necessary for chemistry, biology, and engineering to produce biosensors, their components, transducing systems, and immobilization methods. Biosensors are research instruments that turn an electrical signal into a biological reaction. In general, biosensors need to be extremely precise, autonomous, and versatile to physical parameters such as $\mathrm{pH}$ and temperature. Cammann coined the word "biosensor", and IUPAC presented its concept.

Biosensors categorize compounds into three classes depending on their mechanisms: a biocatalytic group of enzymes; bio affinity category of antibodies and nucleic acids; and microbes [1].

\section{TYPES OF BIOSENSORS}

The Clark and Lyons pioneers introduced Biosensors in the 1960s. The enzyme-based and tissue-based biosensors, immunosensors, DNA biosensors, and thermal-based piezoelectric biosensor forms are used.

Updike and Hicks published in 1967 the first enzyme-based sensor. The immobilization approaches, i.e. enzyme adsorption, ionic bonding, ionic bonding, or covalent bonding is planned for enzyme biosensors. Oxidoreductases, polyphenol oxidases, peroxidases, and amine oxidases are the most common enzymes used for this function.

Davies also developed the first microbe or cell sensor. Material for tissue-based sensors is extracted from plant and animal sources. The value research may be a cycle barrier or a substratum. For the determination of amino acid arginine Rechnitz has created the first tissue-dependent sensor. Membranes, chloroplasts, mitochondria, and microsomes were created from organelle-based sensors. However, the sensitivity for this biosensor form was high, but the detection period was longer, and the specificities were the.

Antibodies, that is to say, bundle to pathogenic or toxin antibody or associate with components of host immune systems, are extremely affinous to their respective antigens, have been identified as immunosensors [2].

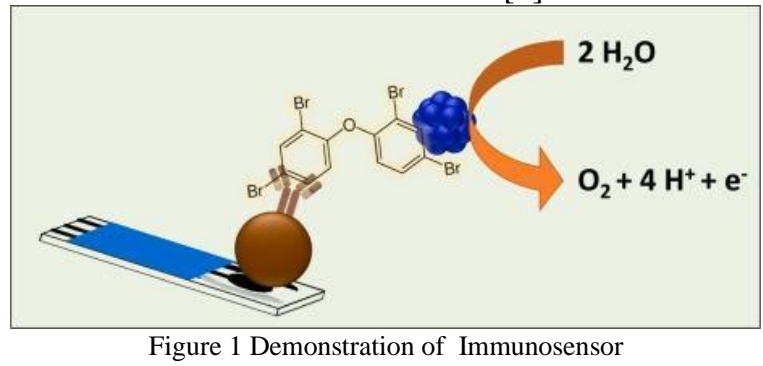

The creation of genetically engineered biosensors has been supported by the production of green fluorescent proteins and subsequent autofluorescent protein (AFP) variants and genetic fusion reporters. This is a user-friendly form of biosensor that is simple to build, manage, and pass to cells. Another example of this is the single-chain FRET biosensor. They are made up of a pair of AFPs and can pass fluorescence resonance energy together. Different methods of controlling the changes in the strength, ratio, or lifetimes of AFPs in Förster Resonance Energy Transfer (FRET) Signals may be used. The synthetic chemical processes followed by the enzyme labeling with synthetic fluorophores enable the manufacture of peptides and protein biosensors. Their autonomy from genetically encoded BFAs improves the signal-to-noise link and reaction responsiveness of the chemical scanners and the photoactivable community, which allows it simpler to monitor the target operation and have an attractive option.

\section{APPLICATIONS OF BIOSENSORS}

In several areas, biosensors were introduced, such as the food industry, medical, aquatic, etc., which give greater stability and flexibility than the normal methods.

Quality and health, management of food items, and manufacturing are a challenging job in the food processing industries. Orthodox chemistry and spectroscopy methods have drawbacks, expense, and time-consuming due to human exhaustion. For Food Industry, alternative steps to authenticate and track food items in a cost-effective manner with accurate and reliable measuring are ideal. Sweeteners, which cause undesired illness including dental cavities, cardiovascular disorders, overweight and type- 2 diabetes, are one of the 
common food additives commonly used today. Artificial sweeteners are believed to be addictive and to encourage us unwittingly ingesting more high-energy items, inadvertently contributing to weight gain. Its identification and analysis are therefore of the utmost importance. Traditionally, ion chromatographic approaches are difficult and laborious in discriminating between two forms of sweeteners.

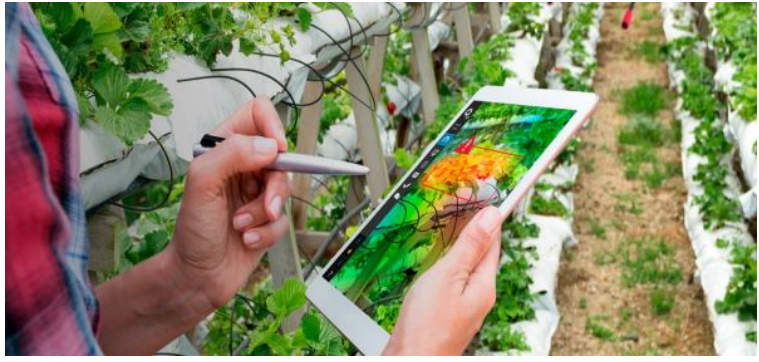

Figure 2 Biosensors and food sustainability

Process protection and product quality are important in the fermentation industries. Therefore, it is crucial to create, upgrade, and sustain bioreactors at the highest effectiveness to track the fermentation cycle effectively. To indirectly assess process parameters, biosensors may be used to track the presence of ingredients, plants, enzymes, antibodies, or plant by-products. Thanks to its easy instrumentation, enormous selectivity, low price, and clear automation, biosensors specifically monitor the fermentation industry and deliver reproducible tests. Today, a range of commercial biosensors are available; they can detect and are commonly used in China for biochemical parameters.

Biosensors are often used to track shifts in the biochemical composition in the ion exchange retrieval network. E.g., glutamate biosensor was used to perform ion-exchange experiments for the recovery of isoelectric glutamate supernatant liquor. The fermentation method is a Byzantine mechanism with several main variables, often in real-time. To promote rapid optimization and track biological processes, online tracking of essential metabolites is key. Thanks to its usability and fast reaction, biosensor technologies have been strongly attracted by online fermentation monitoring in recent years.

The specific biosensor applications include quantitative cardiac markers in undiluted serum, endothelin mediated heart hypertrophy microfluid impedance monitoring, an immunosensor system for the therapeutic immunophenotyping of acute leukemias, the impact of oxazaborolidines on immobilized fructosyltransferase in dental diseases.

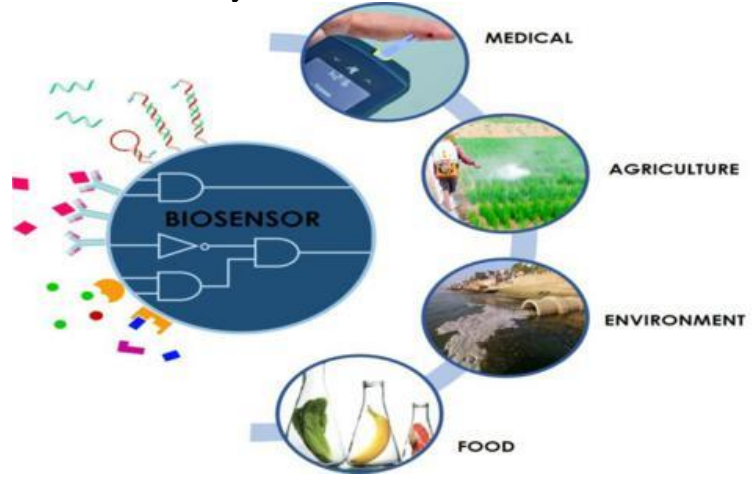

Figure 3 Application of Biosensors
In drug-detection schemes, fluorescent biosensors are used to classify medicines by high-performance, high-containment screening methods, post-screening hits review, and lead optimization. The fluorescent biosensors are effectively used to identify biomarkers throughout the molecular and clinical diagnosis, to track disease progression and patient reaction/therapies, for intravital imaging, and for photography leading These are considered important instruments for preclinical assessment and clinical testing of therapeutic potentials of candidate drugs [3].

IV. THE NEED FOR MULTIANALYTE IDENTIFICATION BY MINIATURE BIOSENSORS

For complicated disorders, extremely accurate disease indicators are frequently missing forcing the healthcare provider to focus on several indicators to identify fake positive results. Since several disease biomarkers are not sensitive enough to detect a single biomarker, their specificity is restricted and cannot be adequately established. Using to classify an illness favorably. Early detection is necessary for efficient diagnosis and a decreased death risk. In the very broad context of similar molecules, one method must be established that can identify a few biomarkers at a very small concentration. To resolve this problem, many long-term checks for accurate disease detection are also conducted. Healthcare practitioners also rely on numerous, unobtrusive research and recognition tests performed using specific spatialized equipment. It is an unacceptable mechanism that contributes to shortages, costs, and pain. In chronic illnesses such as cancer, this is particularly relevant.

Regrettably, existing sensor technologies do not follow the reliability, selectivity, simultaneous detection, expense, and future usage of limited sample requirements.

Nevertheless, micro family cantilever biosensing technology should meet all the stringent requirements in very limited sample volumes for very delicate and specific multitarget detections. Thanks to their ability as a highly reactive medium for high sensitivity and multiplex identification of chemical and biomolecules, microfabricated cantilever array captors have drawn significant interest over the last decade. Micro cantilevers are electric and mechanical beams fixed in one place much like small dive decks. Such wafers and other items can easily be made from silicone.

In general, biological responsiveness to recognition is accomplished on one side of the surface layer by immobile target receivers of sample molecules. A cantilever beam with immobilized receivers is bent as it is bathed in fluid and goal molecules on the surface. The amount of target molecules on the cantilever region which binds to the unmovable receptors is depending on the distance of the cantilever bending.

The standard measurements are around $100 \mu \mathrm{m}$ in length, 20 $\mu \mathrm{m}$ in width, and $1 \mu \mathrm{m}$ in thickness. Microcantilever is highly responsive displacement sensors, also detecting weaknesses involved in molecular adsorption (in contrast, a human hair's diameter is about $80 \mu \mathrm{m})$. As we can later see, this nanoscale deflection is triggered by adjustments in the adsorption-induced free energy of the cantilever surface tension. A piezoresistive reading technique that enables interaction with a miniature tool will test the bending of the cantilever beam electrically. 
While the detection period can be substantially reduced as cantilevers are generated into arrays for simultaneous detection of several analytes, resulting in tremendous savings of costs and time. High diagnosis sensitivity contributes to early identification, reduction in incidence, symptoms, and levels of mortality for certain challenging conditions. Both such benefits should potentially minimize research expenses and, therefore, prices for patients and healthcare practitioners. Unlabeled biomarkers are identified by Cantilever sensors in fewer manufacturing methods than the testing systems presently usable, such as enzyme-linked immunosorbent assays of proteins or nuclear acid microarrays.

Since micro-fabrication techniques include the mass manufacturing of miniature sensors, the microcantilever arrays are transparent in the phase of creating cost-efficient sensors for biosensing applications with unprecedented sensitivity.

In resonance frequency mode, microcantilever sensors may be controlled where mass loading is observed as shifts in resonance frequency due to molecular adsorption. Several bioanalyses were identified with the resonance frequency test. The resolution of the frequency controls is therefore weak, because of the low consistency element. The frequency damping in liquids [4].

\section{CANTILEVER DEFLECTION SYSTEM'S MECHANICS}

Solids contain subtle friction, which is sometimes referred to as 'superficial free energy' like liquids. Solid-surface atoms are trapped into a structure of gills, close to the fluid molecules in a free-range environment. The propensity to reduce energy (molecular adsorption decreases solid surface free space) often assembles the pins on a firm sheet. If, for example, the surfaces on the opposite surface inert adsorption on molecules on the surface of thin material is mostly limited to one side, a gap is created. The substance is deformed by this unequal surface tension. With a microcantilever laser, this impact can easily be seen. Surface stress $\sigma$ and surface free energy $\gamma$ can be related to the equation of Shuttleworth.

$$
\sigma=\gamma+\frac{d \gamma}{d \varepsilon}
$$

Where surface strain $d \varepsilon$, a ratio of surface area change to a total area is described as

$$
d \varepsilon=\frac{d A}{A}
$$

The second term for liquids is null.

Molecular adsorption results in a change in surface stress and surface-free capacity. Molecular adsorption induces a differential surface tension, resulting in extreme bending. Stoney's Equation relates to the gap between the surface tension $\Delta \sigma$ and the uncontrolled surface with the cantilever deflection $\Delta h$ :

$$
\Delta h=\frac{4(1-v) L^{2}}{E t^{2}}\left(\Delta \sigma_{1}-\Delta \sigma_{2}\right)
$$

In case $\mathrm{v}$ is a Poisson content ratio, $\mathrm{E}$ is a young material (elastic) that is cantilever, while $\mathrm{t}$ and $\mathrm{L}$ are the cantilever thickness and length respectively. The length and thickness of an optimizer allow it to be highly immune to minor energy changes without surface use. Contrary to a sterile surface in a vacuum, adsorbed surfaces are subjected to the ambiance. Molecules entering adsorption locations. The goal molecules adsorb or destroy adsorbed molecules on the surface until they are exposed to objective molecules. Therefore, the change in the measured surface energy of the experiment cannot be readily associated with theoretical values.

\section{PARASITE DEFLECTION SOURCE}

The subsequent deflections are mostly attributed to a mixture of various impacts on the cantilever surfaces. Cantilever deflection can be caused by physical factors such as temperature fluctuations and flow levels, $\mathrm{pH}$, and ionic consents. The lifting agent may also be skewed by secondary effects including adjustments in surface load intensity that result in adsorption, ionized material forming as a consequence of the recipient processing operation or swelling of immobilized receptor materials. So soon as the opposite hand of the lift button is separated, the raise button deflects.

Since cantilever construction can be biomaterial, shifts in thermal expansion coefficients can lead to bending with temperature changes. A lifting system is often bent because of the venturi forces caused by wind. Surface species such as silique oxide and silicon nitride ionize according to the $\mathrm{pH}$ of the solution and can bend as well. The reference cantilever is also turned into arrays (paired with sensor cantilever), to remove effects caused by these objects and the differential mode of deflection is calculated as regards the reference cantilever.

\section{CANTILEVER DEFLECTION E-MONITORING}

An optimized transistor procedure for the metal-oxide field effect (MOSFET) transistor is installed at the base of the transistor. The force from the curvature of the carriers affects the stability of the carriers and the MOSFET drainage present. The invention of the integrated transistor was not thoroughly researched. Through a range of strategies such as variability of optical deflection, embedded transistor semi-conductivity, piezoelectricity, and Piezoresistivity, the rotation of a cantilever beam may be sensitively controlled. The benefits and drawbacks of all the different signal transduction approaches are dependent on the procedure. The beam of light from the tip of the laser cantilever to a responsive position detector during the optical beam deflection is observed by projecting this shift. Although the optical beam deflection process is commonly used in sensor processing, the sensors are not lightweight or economical.

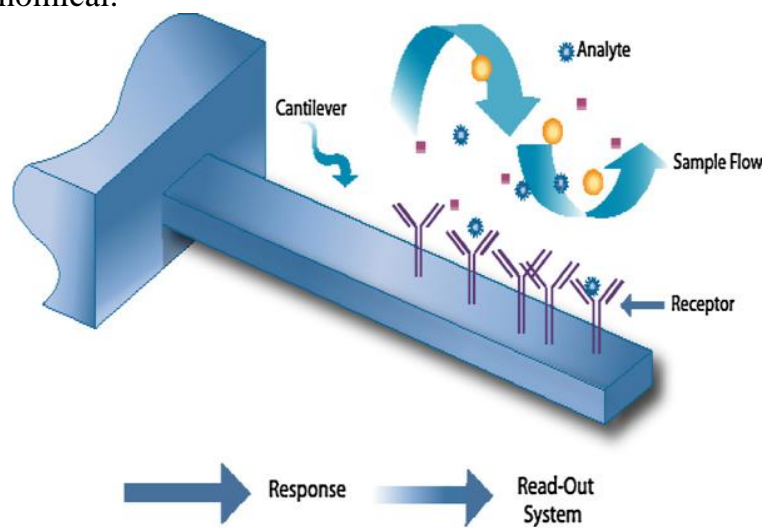

Figure 4 Biosensing using dynamic-mode cantilever sensors 
The resistance of an asymmetrically doped tube differs based on bending with the piezoresistive technique. As a signal transduction tool for biosensor applications, the piezoresistive approach has been widely studied. The piezoresistive technique is consistent with the miniature method. The description of piezo-design cantilever development will be covered in the next segment [5].

\section{PIEZORESISTIVE ARRAYS DESIGN}

Piezoresistivity is the feature of some conducting materials, such as doped silicon, whose susceptibility to electricity depends on the strain applied. The cantilever is built from an integral resistor, which has a piezoresistive property, and its Resistance varies based on the bending of the cantilever, by utilizing a piezoresistive technique to regulate the deflection. A basic electrical calculation will sensitively calculate the degree of the cantilever bending. The normally developed piezo resistors are based on silicon layers doped with boron. The silicon layers of the doped are then separated. The piezoresistive coating of the boom should be mounted on the surface ideally in the area of highest stress to maximize protection. The corresponding relative resistance shifts are directly proportional to the tension and, therefore, to the deflect for a first approximation.

Variation of cantilever resistance is usually measured against a reference raise produced adjacent to the cantilever sensor. The definition of calculation is illustrated in the figure schematically. The reference lens is inert and is used to minimize disturbances from the device, such as variations in temperature. The sensor lift device is layered with a detector layer on one side, that binds specifically with the molecule of interest, as does the reference lift device. Generally, polyethylene glycol is rendered bioinert on the contrary of the functionalized substrate of resist undefined adsorption.

The cantilever begins bent as the molecules attach on the coated surface since the surface tension variations between the two sides of the cantilever are different. Usually, two external resistors link the cantilever sensor in a Wheatstone bridge setup to the reference cantilever. It allows the feedback signal to be reached even where the deflection between the two cantilevers is equal and thus noise is greatly minimized. Fig 2 displays a device for differential calculations of two microfabricated bottles.

A small scale allows the vibrators resilient with a strong resonant frequency which allows them less prone to external vibration. The resistor to silicone is identified by nitride silicone or micro-crystalline crystal. On both sides of the package, the silicon nitride thickness is regulated, and the neutral axis of the package is within the silicon nitride layer rather than the silicon sheet. Such asymmetry means that the resistor is positioned next to one of the surfaces of the cantilever for optimum response.

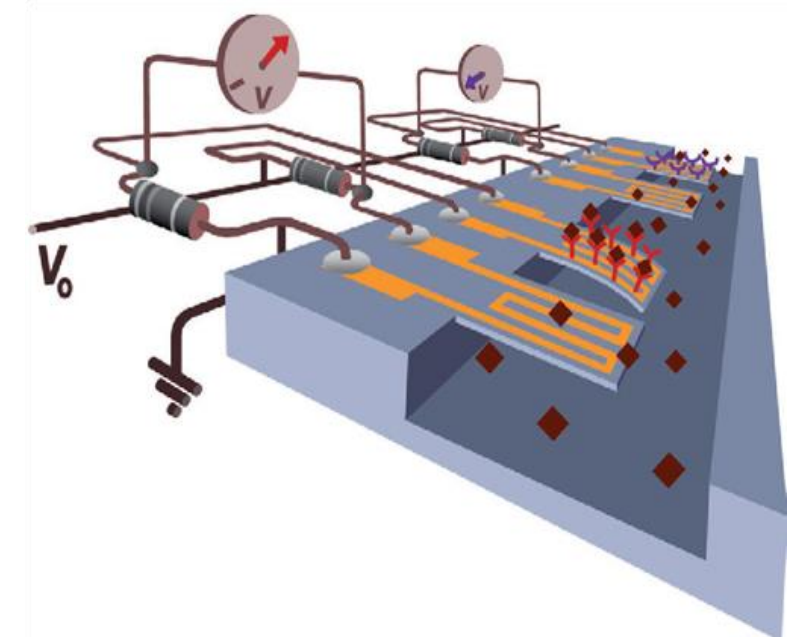

Figure 5 The cantilever calculation theory diagram model. The lift bends and bending are observed as molecules bind to the suspension as a shift of tension within the boost lift. Two cantilevers are often tested at the same moment.

Only the difference signal is registered, and one cantilever serves as a reference

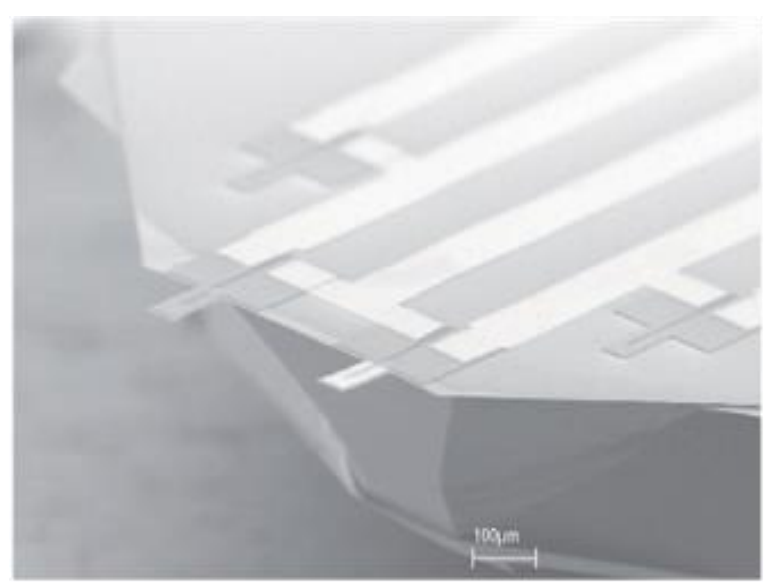

Figure 6 Picture of two silicon lifting devices in which one at the end of the molecules of sulfur is filled with a thin layer of gold for the linking party.

Advanced mechanical devices with measurements of micrometers and embedded components cannot be made utilizing fine mechanics. Instead, methods focused on cleanrooms are used in which a three-dimensional etching of a thin silicon wafer creates the cantilevers. Depositing the silicon nitride compounds with low-pressure organic vapor deposition. Specific geometries and configurations of cantilever structures are readily created and can be combined with liquid handling networks. In figure 3 , is seen in the channel a picture of 32 cantilevers. The canal helps to channel the liquids to the cantilevers under examination. The limited scale of the transducers allows an optimized system that can accommodate liquid and re-read electronics on a few centimeters [6]. 


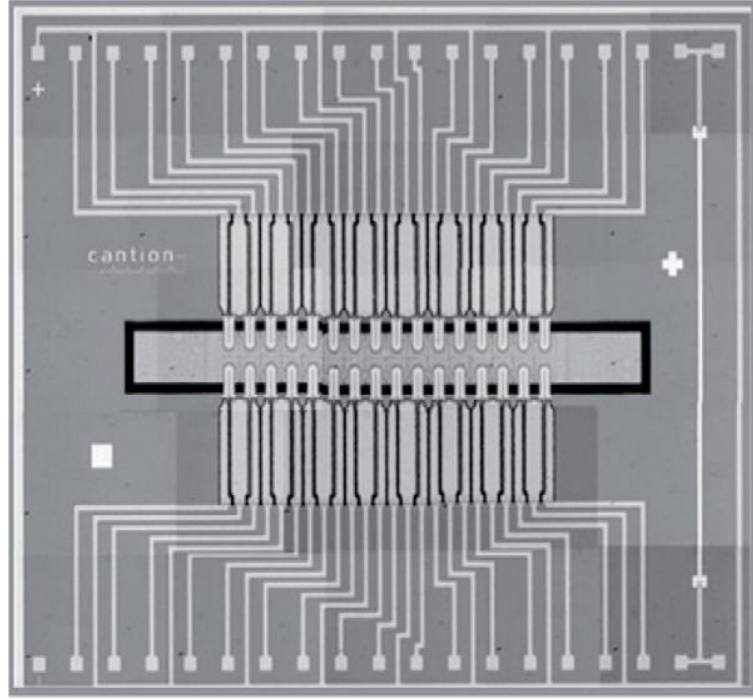

Figure $7 \mathrm{~A}$ set of 32 piezoresistive cantilevers organized in a microfluidic channel.

A cantilever 's strength defines the surface stress response. Therefore, polymerized cantilevers have greater deflection flexibility and are far more versatile. E.g., an epoxy-based polymer SU-8 cantilever can be rendered 40 times softer than silicone. This increases efficiency at once and substantially reduces the cost of production. By spinning out the polymer on to a silicon conveyor wafer, the polymer cantilevers are coated in an anti-sticking coating. The polymer is formed by ultraviolet lithography and subsequent layering and design steps. Inside micrometer-sized tubes, cantilevers may be mounted. The cantilevers can easily be separated with tweezers when processing is complete.
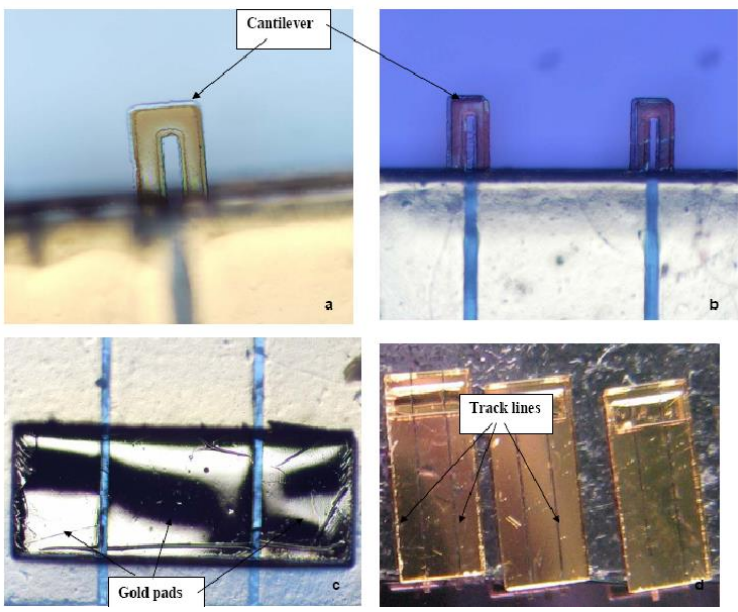

Figure 8 Polymer picture (SU-8) cantilevers with built-in gold resistance. This system is simpler and much easier to produce than comparable silicon products. Polymers also need to be classified with the same durability as silicon, though. (Alicia Johansson's courtesy).

An example of an incorporated gold resistor polymer cantilever appears in fig. 4. Signal-to - noise efficiency is equivalent to the performance for silicon nitride lifters with a single crystal resistor40. The gold resistors are low-noise piezo resistors. The relative gold shift is around 40 times less than single-class silicon resistors and, as candidates for even better piezo resistors, new materials are therefore being explored. The task is to find a soft material when making a big resistance transition. The device will also have minimal electrical noise. The study has been performed on Poly(3,4-ethylene dioxythiophene) (PEDT) and SU-8 doped with $20 \mathrm{~nm}$ of $\mathrm{CO} 2$ particles [2].

\section{BIOSENSORS FOR MICROCANTILEVER}

The chromium acts as a gold binding sheet. For the immobilization of Silicon Oxide cantilevers, silane chemistry may be used. Dip-coating is the favored approach for the immobilization of receptors to obtain a strong grafting density for thiol auto assembled single-layer (SAMs) and organ silane alteration. A monolayer on a gold foil is self-limited by the SAM. Microcantilever beams are particularly sensitive sensors to displacement. Because there is no inherent chemical or biological selectiveness, highly sensitive receptors on the cantilever surface are often immobilized to gain identification selectivity. The selectivity of the recognition is therefore dependent on the selectivity of the receptor study. Many strategies were used to immobilize molecular detection agents of the microcantilever array. Thiol chemistry is a commonly used mechanism by which receptors may be inserted. The ebeam evaporator fills the cups for the first time with chromium ( 2 to $3 \mathrm{~nm}$ ) and gold ( 25 to $30 \mathrm{~nm}$ ).

The organ silane coatings are often of the form of a monolayer but may be multilayered as the formulation is expanded. Any experiment is planned for practical surfaces. By using several sensors in tandem to increasing the sensitivity of microcantilever array detection. For biomedical applications, such improved reliability is especially important. Microcantilever arrays assembled in clusters may also be used to track several biomarkers simultaneously.

Nanomechanical biomolecular identification of cantilever clusters offers tremendous incentives to biosensor marks. A variety of biomolecules were observed with the platform cantilever. DNA strands can be observed as the easiest biosensor example. The thiol chemistry is used on the goldcoated side of the cantilever to immobilize single-stranded DNA molecules (DNA samples). The one-stranded immobilization of DNA results in a broad bending operation. The hybridization and further bending of the cantilever through a current of single-stranded DNA (target DNA). Fig. 5 shows cantilever hybridization with DNA bending. When all the DNA samples are hybridized, the response of the cantilever to hybridization is steady. The same principle is often important to protein identification. Fig . 6 demonstrates cantilever folding to a cantilever utilizing immobilized antibodies as a feature of time, owing to unique C-reactive Protein Adsorption (CRP). 


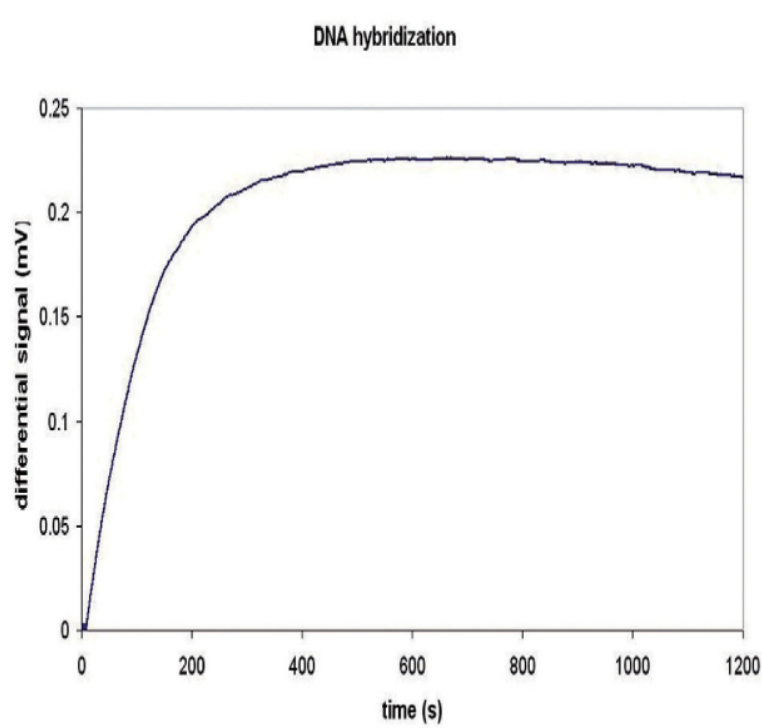

Figure 9 DNA hybridization is observed with a single-stranded DNAimmobilized cantilever. When a single-stranded DNA solution was introduced in the spray, the figure indicates cantilever bending.

The lance is first handled with CRP antibodies in this case. In this case. The association between the antibody and antigens allows the sling to curve. Many biomolecular associations at room temperature are permanent.

Biosensors that were recently installed using a lifting device have been developed. The embedded sensors were used to monitor blood gases, such as blood alcohol, as recovery from a biosensor was extremely difficult. In this situation, the posterior pads were protected with hydrophobic insulation and the gold side of the posterior pads were painted with a thiolated polymer siloxanefluoro alcohol (TSXFA). The TSXFA surface removes alcohol and produces a broad curve. Many polymers with higher alcohol partition feature (OV17 MCP20) have been used as a preferential substrate, for instance, methyl phenyl mercaptopropyl silicone. A transmitter to measure blood alcohol levels in a short-term in vivo device utilizing Ferrell et's optimized re-reading and telemetry cantilevers. In Fig., al. can be seen in fig 7 . Owing to many existing technical shortcomings, in vivo identification by implantable sensors is a huge task. E.g., no highly selective receptors are currently accessible that can function efficiently in vivo. Regenerating the sensor system and preserving the output of the receptors after identification

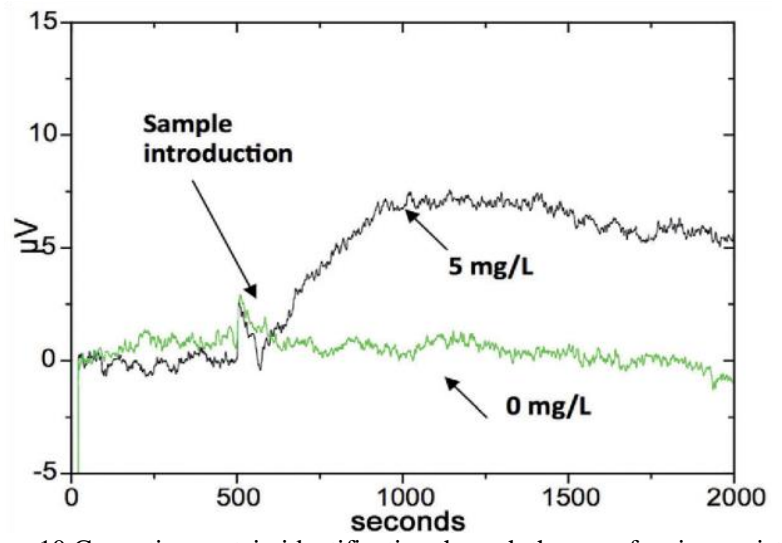

Figure $10 \mathrm{C}$-reactive protein identification through the use of a piezoresistive CRP-antibody immobilized cantilever.

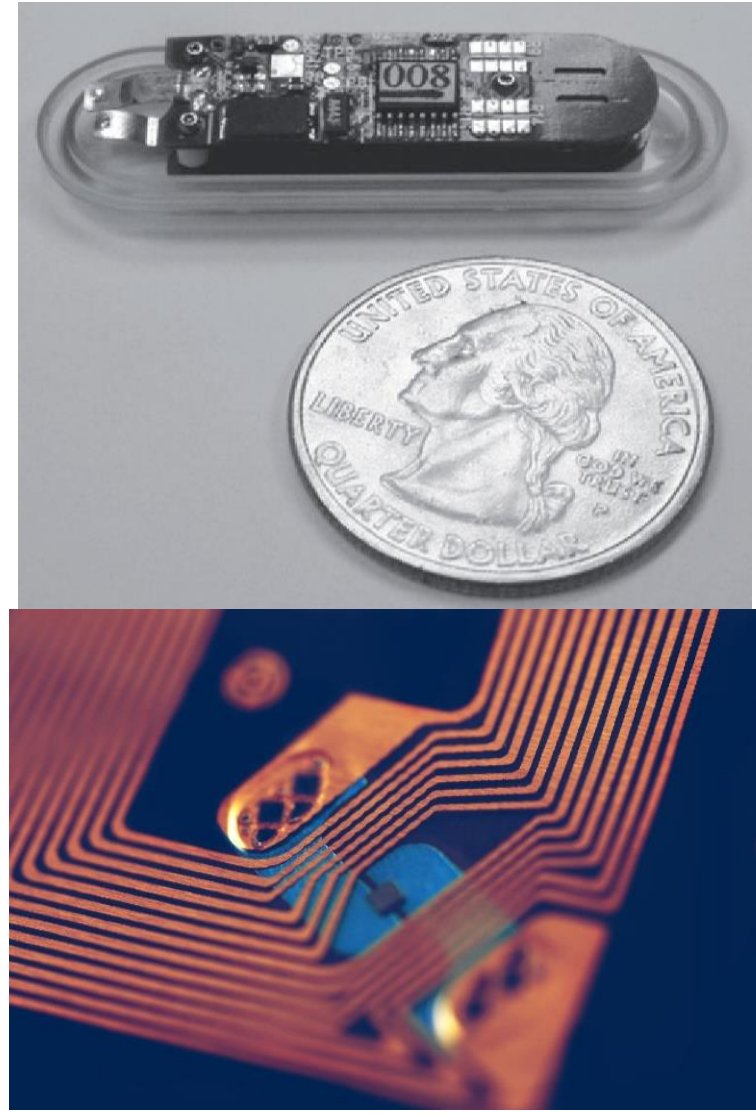

Figure 11 A built-in, portable telemetry implantable tracker for blood gas detection. The figure depicts an implantable capsule closed and open opposite a quarter coin in the United States. In the upper section, a titanium fruit is shown on the left side of the locked capsule as the circular membrane. A membrane filter to partly isolate the analyzer from the interstitial fluid is placed below the frit. The two sets of four microcantilever piezoresistive sensors linked by a series of bridges from Wheatstone are used. The signals are sent to a sigma-delta ADC and thus to a microprocessor with very low strength. This is designed to modulate the transmissions to a similar combination of recipients/computers where details are displayed graphically. In today 's shape, both chemical and body temperature can be transmitted every five minutes for six weeks by the Tele sensor capsule (T.L. Ferrell Courtesy)

Significant issues do need to be treated over long periods in vivo. Potential sensor surface biofouling and the biocompatibility of the chemical interface are both problems for target-analyte adsorption [7].

\section{COMPLICATIONS, CHALLENGES, AND DISCUSSION}

To simplify applications, techniques for sampling, and injection into the sensor arrays are also needed. It is important to research the function of specific lifting parameters, such as the crystalline nature of the coating content. More detail on the cycle of biomolecular adsorption on the cantilever is also required. Experimental findings from our laboratory and laboratories across the globe show that the analysis of several samples from a raw sample with the present technology cannot be accomplished. Electronic readings include developed technologies to develop and produce cantilever clusters. However, in light of the numerous benefits of a microfabricated, several obstacles continue to be addressed until a large audience can be sought. Much because the semiconductor electronics industry has encountered similar 
obstacles, we expect that these problems can be resolved. The design of channel arrays and channel networks in cantilever structures is still under progress. Recipient immobilization in a reproducible manner from device to device on individual cantilevers remains a problem. New reliable strategies of receptor immobilization that can function under a solution reproducibly and maximize receptors on the cantilever. The difference in output characteristics from array to array is not suitable for advertisements because of a variance in the density surface of immobilized receptors. It is important to research the longevity and impact of immobilized receptors on their efficiency. Therefore, before the identification with lifting arrays, it is important to incorporate some basic on-chip sample processing. This on-chip processing can be implemented using currently accessible techniques without growing chip size or power requirements [8].

\section{CONCLUSION}

While unparalleled responsiveness and receptor-based selectivity for microcantilever biological sensors have been seen, output efficiency is not compatible with accurate and replicable receptor immobilization problems. The deposition and immobilization of the specific biological and chemical coatings appear to be a vital issue of continuity and productiveness. There is a range of market uses, spanning from hospitals and food protection to environmental sustainability after these obstacles have been resolved. This is especially valid if many analytes are to be identified.

\section{REFERENCES}

[1] Berger, R., et al., Surface stress in the self-assembly of alkanethiols on gold. 1997. 276(5321): p. 2021-2024.

[2] Boisen, A. and T.J.M.t. Thundat, Design \& fabrication of cantilever array biosensors. 2009. 12(9): p. 32-38.

[3] Butt, H.-J.J.J.o.c. and i. science, A sensitive method to measure changes in the surface stress of solids. 1996. 180(1): p. 251-260.

[4] Chen, G.Y., et al., Adsorption-induced surface stress and its effects on resonance frequency of microcantilevers. 1995. 77(8): p. 3618-3622.

[5] Thundat, T., P. Oden, and R.J.M.T.E. Warmack, Microcantilever sensors. 1997. 1(3): p. 185-199.

[6] Thundat, T., et al., Thermal and ambient-induced deflections of scanning force microscope cantilevers. 1994. 64(21): p. 2894-2896.

[7] Dutta, P., et al., Enantioselective sensors based on antibody-mediated nanomechanics. 2003. 75(10): p. 2342-2348.

[8] Lavrik, N.V., M.J. Sepaniak, and P.G.J.R.o.s.i. Datskos, Cantilever transducers as a platform for chemical and biological sensors. 2004. 75(7): p. 2229-2253. 